Electrical support during outdoor cycling in patients with coronary artery disease: impact on exercise intensity, volume and perception of effort Peer-reviewed author version

HANSEN, Dominique; Soors, An; Deluyker, Valerie; FREDERIX, Ines \& DENDALE, Paul (2018) Electrical support during outdoor cycling in patients with coronary artery disease: impact on exercise intensity, volume and perception of effort. In: ACTA CARDIOLOGICA, 73(4), p. 343-350.

DOI: $10.1080 / 00015385.2017 .1385153$

Handle: http://hdl.handle.net/1942/25012 


\title{
Electrical support during outdoor cycling in patients with coronary artery disease: impact on exercise intensity, volume and perception of effort
}

\author{
Dominique Hansen $^{1,2}$, An Soors ${ }^{1}$, Valerie Deluyker ${ }^{1}$, Ines Frederix ${ }^{1,2,3}$, Paul Dendale ${ }^{1,2}$ \\ ${ }^{1}$ Faculty of Medicine \& Life Sciences, Hasselt University, Agoralaan gebouw D, 3590 Diepenbeek, Belgium \\ ${ }^{2}$ Department of Cardiology/Heart Centre Hasselt, Jessa Hospital, Stadsomvaart 11, 3500 Hasselt, Belgium \\ ${ }^{3}$ Faculty of Medicine \& Health Sciences, Antwerp University, Prinsstraat 13, 2000 Antwerp, Belgium.
}

\section{Corresponding author}

Dominique Hansen, PhD, FESC

Hasselt University

Faculty of Medicine and Life Sciences

REVAL, Rehabilitation Research Center

Agoralaan, Building A

3590, Diepenbeek

Belgium

Dominique.hansen@uhasselt.be

Full text word count: 2640 


\begin{abstract}
Background

Electrical assisted bicycles (EAB's) could be used to overcome barriers and difficulties to outdoor cycling and thus assist in achieving a sufficient physical activity level in coronary artery disease (CAD) patients, but it is unknown whether sufficient exercise intensities and volumes could be elicited during cycling on EAB's. In this study we examined, for the first time, the acute physiological impact of electrical support during outdoor cycling in CAD patients (ISRCTN32238279).
\end{abstract}

\title{
Methods
}

Fifteen CAD patients (13 males), aged $64 \pm 7$ years executed a maximal cardiopulmonary exercise test and afterwards cycled a predefined outdoor route of $10 \mathrm{~km}$, in three different conditions: classical cycling (no support), $\mathrm{EAB}$ with low support ( $\mathrm{EAB}_{\text {low }}$ ) and high support $\left(\mathrm{EAB}_{\text {high }}\right)$. Oxygen uptake $\left(\mathrm{VO}_{2}\right)$ and carbon dioxide output $\left(\mathrm{VCO}_{2}\right)$ was measured continuously by a portable gas-analyzing system. Cycling time was recorded and ratings of perceived exertion (RPE) was assessed at 3 and $7 \mathrm{~km}$.

\section{Results}

Mean $\mathrm{VO}_{2}$ during $\mathrm{EAB}_{\text {high }}\left(1721 \pm 537 \mathrm{ml} \cdot \mathrm{min}^{-1}\right)$ was significantly lower compared to $\mathrm{EAB}_{\text {low }}$ $\left(1890 \pm 619 \mathrm{ml} \bullet \mathrm{min}^{-1}, \mathrm{p}<0.05\right)$, but no differences were found between $\mathrm{EAB}_{\text {low }}$ or classical cycling $(1846 \pm 523 \mathrm{ml} \bullet \mathrm{min}-1) . \mathrm{EAB}_{\text {low }}$ and $\mathrm{EAB}_{\text {high }}$ elicited a sufficient volume and intensity (6.6 \pm 2.0 MET's $\left(74 \pm 6 \% \mathrm{VO}_{2 \text { peak }}\right)$ and $6.0 \pm 1.8 \mathrm{MET}$ 's $\left(68 \pm 7 \% \mathrm{VO}_{2 \text { peak }}\right)$, respectively) to adhere to the guidelines for secondary prevention in CAD. RPE was significantly lower $(\mathrm{p}<0.05)$ during $\mathrm{EAB}_{\text {high }}(9 \pm 2)$, than during $\mathrm{EAB}_{\text {low }}(11 \pm 2)$ or classical cycling $(11 \pm 2)$. 


\section{Conclusion}

Outdoor cycling with electrical support leads to a sufficiently high exercise intensity and volume in CAD patients, and may be considered as an alternative exercise modality.

\section{Key words}

Coronary artery disease; electrical assisted bicycles; exercise intensity; exercise volume

\section{Disclosure statement}

No potential conflict of interest was reported by the authors. 


\section{Introduction}

Cardiovascular disease is one of the main health problems, contributing to $47 \%$ and $40 \%$ of all deaths in Europe and the European Union, respectively [1]. After an acute coronary syndrome, cardiac rehabilitation is recommended by the European Society of Cardiology, the American Heart Association and the American College of Cardiology (Class IB), as part of secondary prevention [2]. In this regard, exercise training is acknowledged to be a core component in these programs, as it is known to decrease cardiovascular morbidity and mortality significantly [3-6]. It is recommended to preferably engage in moderate (3-6 MET) to vigorous ( $>6 \mathrm{MET}$ ) aerobic exercise training, $\geq 3$ times a week and for $>30 \mathrm{~min}$ per session. This translates in a minimum goal of >405 MET-min/week [2]. Despite the proven effectiveness of exercise training in cardiac rehabilitation (improvements in physical fitness and cardiovascular risk profile) [7-9], long-term adherence to exercise prescription is often disappointing. This translates into suboptimal control of physical fitness and cardiovascular risk profile in the long term $[10,11]$. Therefore, novel exercise training strategies/devices that have the potential to increase long-term participation rates are urgently needed.

Electrically assisted bicycles provide patients with an alternative to achieve physical activity recommendations, while at the same time reducing the difficulties with classical outdoor cycling (strong contrary wind, hilly courses) and/or with physical limitations (low physical fitness, orthopedic limitations). However, before such exercise modality can be recommended as part of secondary prevention programs for coronary artery disease (CAD), it should be studied whether cycling with electrical assistance elicits sufficient exercise intensities and volumes to contribute to meeting the international exercise training guidelines.

In healthy subjects, it has been found that cycling with both low and high electrical support reduces exercise intensity and/or volume, when compared to classical cycling [12-18], thus potentially leading to the risk of insufficient exercise intensities and volumes to improve 
cardiovascular health. The acute physiological impact of electrical support during outdoor cycling in CAD patients has not been studied before and thus deserves greater attention.

The primary purpose of this study was to assess whether the exercise intensity and volume of cycling with low and high electrical support meets the exercise standards for secondary prevention in CAD. The secondary goal was to study the effect of low and/or high electrical support during outdoor cycling on ratings of perceived exertion.

\section{Methods}

\section{Study design and patient recruitment}

This study (ISRCTN registry: ISRCTN32238279) was a mono-center, prospective, randomized cross-over clinical trial, run at Jessa hospital $(n=15)$ in Hasselt, Belgium between November 2014 and May 2015 (Figure 1). Patients aged between 50-75 years were eligible for participation when they had recently successfully completed phase II cardiac rehabilitation for CAD and treated medically, with a percutaneous coronary intervention or with coronary artery bypass graft surgery. The main exclusion criteria were (i) symptomatic and/or exercise induced cardiac arrhythmias within the previous six months, (ii) physical disability related to musculoskeletal or neurological problems, (iii) severe pulmonary co-morbidity (COPD GOLD III-IV), (iv) chronic kidney disease stage IV-V (GFR: $<30 \mathrm{ml} \bullet \mathrm{min}^{-1} \bullet 1.73 \mathrm{~m}^{-1}$ ) and (v) patients with a pacemaker, internal cardioverter defibrillator and/or cardiac resynchronization therapy device. All patients provided written informed consent, after the nature and possible consequences of the study were explained, prior to study enrollment. The study was conducted in accordance with the principles stated in the Declaration of Helsinki of the World Medical Association, local and national regulations. The study protocol was approved by the medical ethical committee of Jessa hospital and Hasselt university. 
Study participants completed a maximal cardiopulmonary exercise test (CPET) at completion of their 12-week ambulatory rehabilitation program at Jessa hospital, and a classical bicycle cycling route was completed first after which they were randomly assigned to one of two

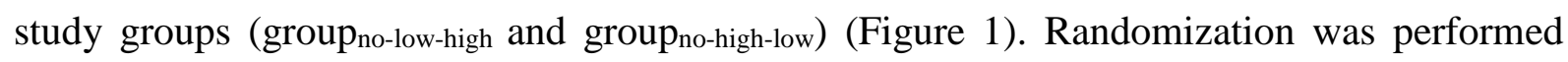

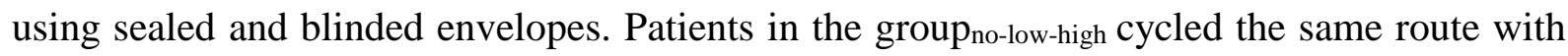
low and high electrical assistance for the second and third time, respectively. Patients in the group $_{\text {no-high-low }}$ cycled the same route with high and low electrical assistance for the second and third time, respectively. Between these routes, a 3-4-day recovery interval was respected.

\section{Subject recruitment and characteristics}

A total of 17 patients agreed to participate in the study (Figure 1). Two patients dropped out after the classical cycling session. There were technical problems with the bicycle for one patient, the other patient had difficulties to cycle without falling. The demographics, comorbidities, medication and initial maximal CPET results of study participants are shown in Table 1 and 2. Included patients (aged $64 \pm 7$ years) were predominantly male $(87 \%(n=13))$ and treated by percutaneous coronary intervention (PCI) $(80 \%(n=12))$ for their CAD.

\section{Initial maximal cardiopulmonary exercise test (CPET)}

Patients performed a maximal CPET $[19,20]$ with a ramping protocol and breath-by-breath gas exchange analysis on an electrically braked cycle ergometer (Jaeger MS-CPX). The test was considered maximal in case of an achieved respiratory gas exchange ratio (RER) >1.1, and/or a ventilatory reserve $\left(\mathrm{VR}\right.$ : VE peak $\left.\bullet \mathrm{MVV}^{-1}\right)>80 \%$. Patients were instructed to cycle at $70 \mathrm{rpm}$, and the test was ended when the patient was no longer able to cycle >59 rpm. Oxygen uptake $\left(\mathrm{VO}_{2}\right)$, minute ventilation $(\mathrm{VE})$ and carbon dioxide output $\left(\mathrm{VCO}_{2}\right)$ were measured continuously and averaged every 10 seconds. The first ventilatory threshold (VT1) was 
defined by V-slope method. Patients with cardiac arrhythmias and/or (silent) myocardial ischemia were excluded from this study.

\section{Experimental procedure}

Two types of bicycles were used: the Montego (classical bicycle) without electrical support, and the E-bike (e.move, Temse, Belgium, model: c300) electrically assisted bicycle with low $\left(\mathrm{EAB}_{\text {low }}\right)$ and high support $\left(\mathrm{EAB}_{\text {high }}\right)$ mode. The electrically assisted bicycle was equipped with a motor located in the hub of the front wheel, providing an additional mechanical support up to $250 \mathrm{~W}$ and a maximal assisted speed of $25 \mathrm{~km} \bullet \mathrm{h}^{-1}$. It could provide electrical assistance with 6 incremental support modes. Electrical assistance was provided up to $12-13 \mathrm{~km} \cdot \mathrm{h}^{-1}$ in mode $1,16-17 \mathrm{~km} \cdot \mathrm{h}^{-1}$ in mode $2,19-20 \mathrm{~km} \cdot \mathrm{h}^{-1}$ in mode $3,22-23 \mathrm{~km} \cdot \mathrm{h}^{-1}$ in mode $4,23-24$ $\mathrm{km} \bullet \mathrm{h}^{-1}$ in mode 5 and $25 \mathrm{~km} \bullet \mathrm{h}^{-1}$ in mode 6 . Each time, the saddle and handlebars were repositioned to suit each subject. Participants were familiarized with the electrically assisted bicycle prior to starting the cycling route, in order to be able to use it appropriately. $\mathrm{EAB}_{\text {low }}$ was defined as support mode 1 and $\mathrm{EAB}_{\text {high }}$ as mode 2 in case patients cycled on average $<16$ $\mathrm{km} \bullet \mathrm{h}^{-1}$ during the classical cycling sessions. $\mathrm{EAB}_{\text {low }}$ was defined as support mode 2 and $\mathrm{EAB}$ high as mode 4 in case patients cycled on average $>16 \mathrm{~km} \bullet \mathrm{h}^{-1}$ during the classical cycling sessions.

Patients cycled on a public track in $\mathrm{x}$. The track had an up-and-down course (102-m height difference) and a length of $10 \mathrm{~km}$. Tests were conducted from 13.00 p.m. to 17.00 p.m.. The weather conditions ranged from $0^{\circ} \mathrm{C}$ to $17^{\circ} \mathrm{C}$, a wind speed between $3 \mathrm{~km} \bullet \mathrm{h}^{-1}$ and $41 \mathrm{~km} \bullet \mathrm{h}^{-1}$, and a rainfall between $0 \mathrm{~mm} \bullet \mathrm{day}^{-1}$ and $8 \mathrm{~mm} \bullet \mathrm{day}^{-1}$. Tests were performed in real traffic, no stop-and-go points were built in the protocol. Patients were instructed to cycle at a selfselected comfortable speed, they did not receive any feedback during or after the cycling 
sessions. One researcher cycled behind the patient for safety reasons and to assist if necessary (in case of mechanical problems).

\section{Outcome measures}

The parameters measured during the cycling sessions were $\mathrm{VO}_{2}, \mathrm{VCO}_{2}, \mathrm{VE}, \mathrm{RER}$, cycling time and ratings of perceived exertion $(\mathrm{RPE}) . \mathrm{VO}_{2}\left(\mathrm{ml} \bullet \mathrm{min}^{-1}\right), \mathrm{VE}\left(\mathrm{ml} \cdot \mathrm{min}^{-1}\right)$ and $\mathrm{VCO}_{2}$ $\left(\mathrm{ml} \cdot \mathrm{min}^{-1}\right)$ were determined (averaged every 10 seconds) by analysis of expired gases by using a mobile Jaeger ergospirometer (Oxycon ${ }^{\mathrm{TM}}$ mobile device). $\mathrm{VO}_{2}$ measurements were referred to the patients' $\mathrm{VO}_{2}$ peak $\left(\% \mathrm{VO}_{2 \text { peak }}\right)$ as deduced from the initial maximal CPET. Exercise intensity was estimated by converting $\mathrm{VO}_{2}$ into MET $\left(1 \mathrm{MET}=3.5 \mathrm{ml} \cdot \mathrm{kg}^{-1} \cdot \mathrm{min}^{-1}\right.$ $\mathrm{VO}_{2}$ ). The mean MET value per cycling session was computed and averaged for classical

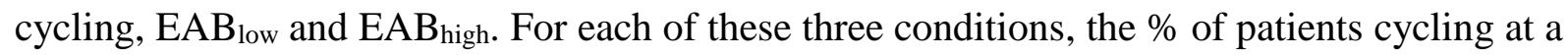
low, moderate and vigorous intensity (defined as <3 MET's, 3-6 METs and >6 METs respectively) was calculated. Cycling time was measured by chronometry (Xonix watch). Total exercise volume was expressed in MET-min by multiplying the mean MET score and the total cycling time (min). Energy expenditure (kcal) was calculated by the following formula: number of $\mathrm{kcal}=$ MET $\mathrm{x}$ weight $(\mathrm{kg}) \mathrm{x}$ cycling session duration (hours). The Borg scale (6-20) was used to assess subjectively reported RPE at two predefined locations (at 3 and $7 \mathrm{~km}$ of cycling route). Only effective cycling periods were included in the final analysis.

\section{Statistical analysis}

Data analysis was performed using SPSS v. 22. The Shapiro-Wilk test was used to assess normality. Nonparametric tests were used instead of parametric statistics in case assumptions for normality were violated. Repeated measures ANOVA (parametric) or Friedman's ANOVA (non-parametric) compared multiple dependent means. If significant effects were 
observed, post-hoc contrasts with Bonferroni corrections were performed to determine which conditions differed. The significance level for tests was 2-sided $\alpha$ of 0.05 . Continuous variables were reported as mean $\pm \mathrm{SD}$, categorical variables as $\%(\mathrm{~N})$. Since this was the first study to examine the acute physiological impact of electrical support during cycling in CAD patients, it was difficult to execute an a priori sample size calculation. However, it was decided to execute an interim sample size calculation when nine subjects were examined (who were studied before January 2015), based on caloric expenditure. During this calculation, it was found that $>14$ subjects had to be included in the present study to be able to detect significant differences in caloric expenditure between different cycling conditions $(\mathrm{p}<0.05$, two-tailed, power $\alpha>0.80$ ). For these calculations, GPower v 3.1 was used. 


\section{Results}

\section{Exercise intensity}

There was a significant main effect of level of electrical support on mean $\mathrm{VO}_{2}(\mathrm{p}=0.018$, Table 3) during cycling. Post-hoc contrasts revealed that the mean $\mathrm{VO}_{2}\left(1846 \pm 523 \mathrm{ml} \cdot \mathrm{min}^{-1}\right)$ during classical cycling sessions were not different from mean $\mathrm{VO}_{2}\left(1890 \pm 619 \mathrm{ml} \cdot \mathrm{min}^{-1}\right)$ during $\mathrm{EAB}_{\text {low }}, \mathrm{p}=1.00$. Mean $\mathrm{VO}_{2}$ during $\mathrm{EAB}_{\text {low }}$ was however higher, compared to $\mathrm{EAB}_{\text {high }}$ $\left(1721 \pm 537 \mathrm{ml} \cdot \mathrm{min}^{-1}, \mathrm{p}=0.038\right)$, indicating a difference in exercise intensity.

There was a significant main effect of level of electrical support on mean MET's during cycling ( $\mathrm{p}=0.016$, Table 3). Post-hoc contrasts showed significant greater MET's during $\mathrm{EABl}_{\text {ow }}\left(6.6 \pm 2.0 \mathrm{MET}^{\prime} \mathrm{s}\right)$, as opposed to $\mathrm{EAB}_{\text {high }}(6.0 \pm 1.8 \mathrm{MET}$ 's, $\mathrm{p}=0.027)$.

During classical cycling, $0 \%(n=0)$ of patients performed in the low, $40 \%(n=6)$ in the moderate and $60 \%(n=9)$ in the vigorous exercise intensity zones. During $\mathrm{EAB}_{\text {low }}$, the respective percentages were $0 \%(n=0), 47 \%(n=7)$ and $53 \%(n=8)$. During $\mathrm{EAB}_{\text {high, }}$ the respective percentages were $0 \%(n=0), 67 \%(n=10)$ and $33 \%(n=5)$.

\section{Cycling time}

Total cycling time differed significantly between the three conditions $(p<0.001$, Table 3$)$. Post-hoc contrasts revealed the total cycling time to be smaller $(\mathrm{p}<0.05)$ during $\mathrm{EAB}_{\text {high }}$ $(31 \pm 4.7 \mathrm{~min})$ compared to $\mathrm{EAB}_{\text {low }}(35 \pm 5.3 \mathrm{~min})$ and classical cycling $(37 \pm 6.5 \mathrm{~min})$.

\section{Energy expenditure}

Total caloric expenditure differed significantly between conditions $(\mathrm{p}<0.001$, Table 3$)$. During $\mathrm{EAB}_{\text {high }}$ energy expenditure was lower $(249 \pm 53 \mathrm{kcal}, \mathrm{p}<0.05)$, when compared to $\mathrm{EAB}_{\text {low }}(301 \pm 57 \mathrm{kcal})$ or classical cycling $(312 \pm 45 \mathrm{kcal})$. 


\section{Ratings of perceived exertion}

RPE was significantly different between conditions $(\mathrm{p}=0.001$, Table 3$)$, and lower $(\mathrm{p}<0.05)$ during $\mathrm{EAB}_{\text {high }}(9 \pm 2)$, when compared to $\mathrm{EAB}_{\text {low }}(11 \pm 2)$ and classical cycling $(11 \pm 2)$. 


\section{Discussion}

In this study, it was investigated for the first time whether outdoor electrically assisted cycling was able to elicit exercise intensities and volumes that adhere to the international exercise training guidelines for secondary prevention in CAD. It was shown that sufficient exercise intensities and volumes were elicited during cycling, even with high electrical support, and could thus be considered as an alternative exercise modality to stimulate outdoor physical activity in CAD patients.

The elicited exercise intensity during $\mathrm{EAB}_{\text {high }}$ was lower (68\% of $\mathrm{VO}_{2 \text { peak }}, 6.0 \pm 1.8$ MET's) as opposed to $\mathrm{EAB}_{\text {low }}\left(74 \%\right.$ of $\mathrm{VO}_{2 \text { peak }}, 6.6 \pm 2.0 \mathrm{MET}$ 's) but was still sufficiently high to adhere to international exercise standards (3-6 MET) for secondary prevention in CAD patients. $60 \%$ $(n=9)$ of CAD patients during classical cycling achieved a mean intensity of >6.0 MET's. During $\mathrm{EAB}_{\text {low }}$ and $\mathrm{EAB}_{\text {high, }}$ 53\% (n=8) and 33\% (n=5), respectively, met the standards for vigorous-intense exercise ( $>6.0 \mathrm{MET}$ 's). The belief that electrical assistance during cycling should be avoided in exercise training of CAD patients should thus be reconsidered. Furthermore, the findings in this study showed that high electrical support during cycling led to a significant reduction in RPE $(9 \pm 2, \mathrm{p}=0.007)$ and could thus lower barriers for the least-fit patients, by assisting for hilly environments or strong contrary winds, but also for (earlier onset of) fatigue. It is thus speculated, although it remains to be shown in subsequent studies, that the use of EAB's may lead to enhanced adherence to physical activity recommendations and greater cardiovascular disease risk control on the long term in CAD patients.

These findings are well in line with previous studies in which healthy participants were studied. Gojanovic et al. compared the physiological response during classical cycling, and during $\mathrm{EAB}_{\text {low }}$ and $\mathrm{EAB}_{\text {high }}$ in 18 sedentary subjects [12]: during $\mathrm{EAB}_{\text {low }}$ and $\mathrm{EAB}_{\text {high }}$ cycling exercise was less intense, when compared to classical cycling. Louis et al. compared the aforementioned three cycling modes within 10 trained and 10 untrained subjects and also 
observed an exercise intensity reduction during electrical support [13]. Similar differences were found in other studies $[16,17]$. Contrary to these findings, we only found a significant exercise intensity reduction during $\mathrm{EAB}_{\text {high. }}$ Exercise intensity was similar between classical cycling and $\mathrm{EAB}_{\text {low in }}$ our study. Given the fact that an electrically assisted bicycle on average weighs $13 \mathrm{~kg}$ more than a conventional bicycle, the electrical support in $\mathrm{EAB}_{\text {low }}$ could have been insufficient to compensate for this increased mass, hence resulting in similar exercise intensities. Our results remain thus to be verified in a design in which an electrically assisted bike would be used during the classical cycling condition. On the other hand, CAD patients may ask their treating cardiologist whether they should ride a conventional bike or on an electrical assisted bike with electrical support in order to maintain their cardiorespiratory fitness, which is examined in the present study.

Cycling time and hence energy expenditure (as a derivative of both exercise intensity and cycling time) were significantly different between the three cycling conditions $(\mathrm{p}<0.001)$. Post-hoc contrasts yielded only differences between $\mathrm{EAB}_{\text {high }}$ and other cycling conditions. Total energy expenditure was similar during classical cycling and $\mathrm{EAB}_{\text {low. }}$ It thus follows that patients using the $\mathrm{EAB}_{\text {high }}$ need to prolong their cycling time by $20 \%$, to achieve a comparable total caloric expenditure, as opposed to classical cycling or $\mathrm{EAB}_{\text {low. }} \underline{\text { However, even though }}$ compensations in total energy expenditure can be achieved by cycling longer when receiving (greater) electrical support, a lower exercise intensity remains present. It therefore remains to be studied whether similar clinical benefits of cycling can be achieved when electrical support is offered during a physical activity programme (of several weeks to months) in patients with $\underline{\mathrm{CAD}}$.

The present study faces some methodological strengths and limitations. To the authors' knowledge, this study was the first to investigate the acute physiological impact of electrical support during cycling in CAD patients. Study participants also cycled outdoor, thus 
simulating real-life conditions. The continuous registration of $\mathrm{VO}_{2}$ by analysis of expired gases during the cycling sessions provided a direct measure of exercise intensity and volume. However, the weather conditions were different between the cycling conditions, possibly biasing the results, but obviously could not be controlled by the investigators. For example, worse or better weather conditions (especially rain and wind) could elevate or lower the metabolic demand and/or perception of effort to complete the cycling route.

In conclusion, outdoor cycling with electrical support leads to sufficiently high exercise intensities and volumes in CAD patients, and may be considered as an alternative exercise modality. When selecting high electrical support during cycling, it is advised to prolong the cycling session to achieve similar caloric expenditures, as opposed to classical cycling or cycling with low electrical support.

\section{Acknowledgements}

We would like to thank Kim Bonné and Toon Alders for their assistance in patient recruitment and inclusion.

\section{Source of funding}

This work was supported by an unrestricted grant from Heart Centre Hasselt, Belgium. 


\section{References}

1. European Heart Network Web site [Internet]. Brussels (Belgium): European Heart Network. Available form: http://www.ehnheart.org/cvd-statistics.html.

2. Piepoli M, Corra U, Adamopoulos S, et al. Secondary prevention in the clinical management of patients with cardiovascular diseases. Core components, standards and outcome measures for referral and delivery. A Policy Statement from the Cardiac Rehabilitation Section of the European Association for Cardiovascular Prevention \& Rehabilitation. Endorsed by the Committee for Practice Guidelines of the European Society of Cardiology. Eur J Prev Cardiol. 2014;21:664-681.

3. Rauch B, Davos CH, Doherty P. et al. The prognostic effect of cardiac rehabilitation in the era of acute revascularisation and statin therapy: A systematic review and meta-analysis of randomized and non-randomized studies - The Cardiac Rehabilitation Outcome Study (CROS). Eur J Prev Cardiol. 2016;23:1914-1939.

4. Lewinter C, Doherty P, Gale CP, et al. Exercise-based cardiac rehabilitation in patients with heart failure: a meta-analysis of randomised controlled trials between 1999 and 2013. Eur J Prev Cardiol. 2015;22:1504-1512.

5. Sibilitz KL, Berg SK, Tang LH, et al. Exercise-based cardiac rehabilitation for adults after heart valve surgery. Cochrane Database Syst Rev. 2016;3:CD010876.

6. Lane R, Ellis B, Watson L, et al. Exercise for intermittent claudication. Cochrane Database Syst Rev. 2014;7:CD000990.

7. Lin X, Zhang X, Guo J, et al. Effects of Exercise Training on Cardiorespiratory Fitness and Biomarkers of Cardiometabolic Health: A Systematic Review and Meta-Analysis of Randomized Controlled Trials. J Am Heart Assoc. 2015;4:e002014. 
8. Murtagh EM, Nichols L, Mohammed MA, et al. The effect of walking on risk factors for cardiovascular disease: an updated systematic review and meta-analysis of randomised control trials. Prev Med. 2015;72:34-43.

9. Pattyn N, Cornelissen VA, Eshghi SR, et al. The effect of exercise on the cardiovascular risk factors constituting the metabolic syndrome: a meta-analysis of controlled trials. Sports Med. 2013;43:121-133.

10. Hansen D, Dendale P, Raskin A, et al. Long-term effect of rehabilitation in coronary artery disease patients : randomized clinical trial of the impact of exercise volume. Clin Rehabil. 2010;24:319-327.

11. Frederix I, Van Driessche N, Hansen D, et al. Increasing the medium-term benefits of hospital-based cardiac rehabilitation by physical activity telemonitoring in coronary artery disease patients. Eur J Prev Cardiol. 2015;22:150-158.

12. Gojanovic B, Welker J, Iglesias K, et al. Electric Bicycles as a New Active Transportation Modality to Promote Health. Med Sci Sports Exerc. 2011;43:2204-2210.

13. Louis J, Brisswalter J, Morio C, et al. The electrically assisted bicycle: an alternative way to promote physical activity. Am J Phys Med Rehabil. 2012;91:931-940.

14. Simons M, Van Es E, Hendriksen I. Electrically assisted cycling: a new mode for meeting physical activity guidelines? Med Sci Sports Exerc. 2009;41:2097-2102.

15. Theurel J, Theurel A, Lepers R. Physiological and cognitive responses when riding an electrically assisted bicycle versus a classical bicycle. Ergonomics. 2012;55:773-781.

16. Berntsen S, Malnes L, Langåker A, et al. Physical activity when riding an electric assisted bicycle. Int J Behav Nutr Phys Act. 2017;14:55.

17. LA Salle DT, Shute R, Heesch M, et al. Demands of Simulated Commuting Using an Electrically Assisted Bicycle. Int J Exerc Sci. 2017;10:454-464. 
18. De Geus B, Kempenaers F, Lataire P, et al. Influence of electrically assisted cycling on physiological parameters in untrained subjects. Eur J Sports Sci. 2013;13:290-294.

19. Ross RM. ATS/ACCP Statement on Cardiopulmonary Exercise Testing. Am J Respir Crit Care Med. 2003;167:211-277.

20. Fletcher GF, Ades PA, Kligfield P, et al. Exercise Standards for Testing and Training: A Scientific Statement From the American Heart Association. Circulation. 2013;128:873934. 


\section{Figure Captions}

Figure 1. Study flowchart. EAB low: electrically assisted cycling with low support, EAB high: electrically assisted cycling with high support.

Figure 2. Percentage of route cycled at 3-6 METs or $>6$ METs for each subject separately

$\underline{\text { A. Percentage of route cycled between 3-6METs for each subject }}$

B. Percentage of route cycled $>6 \mathrm{MET}$ for each subject

EAB low: electrically assisted cycling with low support, EAB high: electrically assisted cycling with high support. 
Table 1. Demographics, co-morbidities and medication of study participants.

\begin{tabular}{|c|c|c|c|c|c|}
\hline \multicolumn{2}{|c|}{ Demographics } & \multicolumn{2}{|l|}{ Co-morbidities } & \multicolumn{2}{|c|}{ Medication } \\
\hline \multirow[t]{2}{*}{ Age } & $64 \pm 7$ & Blood pressure & & On Beta blocker & $67 \%(10)$ \\
\hline & & Normal $(S B P<140 \mathrm{mmHg}$ and $D B P<$ & & & \\
\hline \multirow[t]{2}{*}{ Gender } & & $90 \mathrm{mmHg})$ & $67 \%(10)$ & On Statin & $93 \%(14)$ \\
\hline & & AHT Grade I (SBP 140-159 mmHg & & & \\
\hline \multirow[t]{2}{*}{ Male } & $87 \%(13)$ & and/or DBP 90-99 $\mathrm{mmHg}$ ) & $20 \%(3)$ & On ACE-inhibitor/ARB & $73 \%(11)$ \\
\hline & & AHT Grade II (SBP 160-179 mm Hg & & & \\
\hline \multirow[t]{2}{*}{ Female } & $13 \%(2)$ & and/or DBP 100-109 mm Hg) & $0 \%(0)$ & On Anti-platelet therapy & \\
\hline & & AHT Grade III (SBP $\geq 180 \mathrm{mmHg}$ & & & \\
\hline \multirow[t]{2}{*}{ CAD clinical presentation } & & and/or $D B P \geq 110 \mathrm{mmHg}$ ) & $13 \%(2)$ & $D A P T$ & $60 \%(9)$ \\
\hline & & $\begin{array}{l}\text { Diabetes mellitus }(\mathrm{HbA} 1 \mathrm{c}>6.5 \% \text { and } \\
\text { fasting glucose }>126 \mathrm{mg} / \mathrm{dl} \text { or }\end{array}$ & & & \\
\hline STEMI & $27 \%(4)$ & random glucose $>200 \mathrm{mg} / \mathrm{dl}$ ) & $13 \%(2)$ & Anti-platelet monotherapy & $40 \%(6)$ \\
\hline NSTEMI & $7 \%(1)$ & Smoking & & On oral anti-diabetics & $7 \%(1)$ \\
\hline$U A$ & $40 \%(6)$ & No & $47 \%(7)$ & On Insulin & $7 \%(1)$ \\
\hline$S A$ & $27 \%(4)$ & Current smoker & $20 \%(3)$ & On Diuretics & $7 \%(1)$ \\
\hline No of vessels involved & & Prior smoker & $33 \%(5)$ & On Anti-arrhytmics & $7 \%(1)$ \\
\hline 1 & $60 \%(9)$ & Hyperlipidemia & $73 \%(11)$ & & \\
\hline$>1$ & $40 \%(6)$ & Weight & & & \\
\hline Vessels involved & & Normal (BMI 18.5-24.9 kg/m2) & $27 \%(4)$ & & \\
\hline$R A C$ & $47 \%(7)$ & Overweight (BMI 25-29.9 kg/m2) & $47 \%(7)$ & & \\
\hline$L A D$ & $67 \%(10)$ & Obesity Grade I (BMI $30-34.9$ kg/m2) & $27 \%(4)$ & & \\
\hline$c x$ & $47 \%(7)$ & Obesity Grade II (BMI 35-39.9 kg/m2) & $0 \%(0)$ & & \\
\hline Therapy for CAD & & Obesity Grade III (BMI $\geq 40 \mathrm{~kg} / \mathrm{m} 2)$ & $0 \%(0)$ & & \\
\hline $\mathrm{PCl}$ & $80 \%(12)$ & Familial history & $60 \%(9)$ & & \\
\hline$C A B G$ & $20 \%(3)$ & Peripheral arterial disease & $20 \%(3)$ & & \\
\hline
\end{tabular}

AHT: arterial hypertension, ARB: angiotensin receptor blocker, CAD: coronary artery disease, DAPT: dual anti-platelet therapy, STEMI:

ST-elevation myocardial infarction, NSTEMI: non-ST elevation myocardial infarction, UA: unstable angina, SA: stable angina, No: number, RCA: right coronary artery, LAD: left anterior descendens artery, $\mathrm{Cx}$ : circumflex artery, PCI: percutaneous coronary intervention, CABG: coronary artery bypass grafting.

Normal blood pressure (SBP < $140 \mathrm{mmHg}$ and DBP < $90 \mathrm{mmHg}$ ), AHT Grade I (SBP 140-159 mmHg and/or DBP 90-99 mmHg), AHT Grade II (SBP 160-179 mmHg and/or DBP 100-109 mmHg), AHT Grade III (SBP $\geq 180 \mathrm{mmHg}$ and/or DBP $\geq 110 \mathrm{mmHg}$ ). Diabetes mellitus is defined as HbA1c $>6.5 \%$ and fasting glucose $>126 \mathrm{mg} / \mathrm{dl}$ or random glucose $>200 \mathrm{mg} / \mathrm{dl}$. Normal weight (BMI $18.5-24.9 \mathrm{~kg} / \mathrm{m} 2)$, overweight (BMI 25-29.9 kg/m2), obesity Grade I (BMI 30-34.9 kg/m2), obesity Grade II (BMI 35-39.9 kg/m2), obesity Grade III (BMI $\geq 40$ $\mathrm{kg} / \mathrm{m} 2$ ). 
Table 2. Baseline maximal cardiopulmonary exercise test results of study participants.

\begin{tabular}{|cccc|}
\hline & Mean & SD & Range \\
RERmax & 1.2 & 0.1 & $1.1-1.3$ \\
HRrest $(\mathrm{bpm})$ & 80 & 13 & $56-113$ \\
HRmax $(\mathrm{bpm})$ & 132 & 20 & $94-155$ \\
DBPrest $(\mathrm{mmHg})$ & 79 & 17 & $55-121$ \\
DBPmax $(\mathrm{mmHg})$ & 92 & 20 & $58-146$ \\
SBPrest $(\mathrm{mmHg})$ & 124 & 26 & $97-186$ \\
SBPmax $(\mathrm{mmHg})$ & 169 & 23 & $136-217$ \\
VO2 peak $(\mathrm{ml} / \mathrm{min})$ & 1,971 & 620 & $1,211-3,388$ \\
VO2 peak $(\mathrm{ml} / \mathrm{min} / \mathrm{kg})$ & 24 & 7 & $16-39$ \\
HRmax $(\% \mathrm{pred})$ & 84 & 12 & $61-103$ \\
Watt $(\mathrm{W})$ & 173 & 68 & $93-330$ \\
VT1 $(\mathrm{ml} / \mathrm{min})$ & 1,432 & 414 & $734-2,123$ \\
VE peak $(\mathrm{l} / \mathrm{min})$ & 85 & 27 & $51-150$ \\
VCO2 peak $(\mathrm{ml} / \mathrm{min})$ & 2,382 & 798 & $1,436-4,324$ \\
& & & \\
\hline
\end{tabular}

RER: respiratory exchange ratio, HR: heart rate, DBP: diastolic blood pressure, SBP: systolic blood pressure, VO2 peak: peak oxygen consumption, VT: ventilator threshold, VE: minute ventilation, VCO2: carbon dioxide output. 
Table 3. Physiological variables during the cycling tests with the classical bike, the electrically assisted bicycle with low support and high support respectively.

\begin{tabular}{|c|c|c|c|c|c|c|c|c|c|c|}
\hline & \multicolumn{2}{|c|}{ Classical bicycle } & \multicolumn{2}{|c|}{ EAB low } & \multicolumn{2}{|c|}{ EAB high } & \multirow[b]{2}{*}{ Poverall } & \multirow[b]{2}{*}{$P$ No vs $L$} & \multirow[b]{2}{*}{$P$ No vs $\mathrm{H}$} & \multirow[b]{2}{*}{$P$ Lvs $\mathrm{H}$} \\
\hline & Mean & SD & Mean & SD & Mean & SD & & & & \\
\hline VO2 mean (ml/min) & 1,846 & 523 & 1,89 & 619 & 1,721 & 537 & .018 & 1.0 & .204 & .038 \\
\hline$\%$ VO2 peak & 73 & 4.6 & 74 & 6.2 & 68 & 7.1 & .012 & 1.0 & .091 & .029 \\
\hline METs & 6.4 & 1.6 & 6.6 & 2.0 & 6.0 & 1.8 & .016 & 1.0 & .201 & .027 \\
\hline Cycling time (min) & 37 & 6.5 & 35 & 5.3 & 31 & 4.7 & $<.001$ & .301 & $<.001$ & $<.001$ \\
\hline MET-minutes & 230 & 24 & 222 & 36 & 183 & 36 & $<.001$ & 1.0 & $<.001$ & $<.001$ \\
\hline Kcal & 312 & 45 & 301 & 57 & 249 & 53 & $<.001$ & 1.0 & $<.001$ & $<.001$ \\
\hline RPE (Borg scale 6-20) & 11 & 2 & 11 & 2 & 9 & 2 & .001 & .486 & .007 & .014 \\
\hline $\mathrm{VE}(\mathrm{I} / \mathrm{min})$ & 60.7 & 16.0 & 63.9 & 20.9 & 55.0 & 16.8 & .006 & .463 & .168 & .018 \\
\hline $\operatorname{VCO} 2(\mathrm{ml} / \mathrm{min})$ & 1,742 & 531 & 1,734 & 569 & 1,542 & 496 & .003 & 1.0 & .028 & .031 \\
\hline RER & 0.9 & 0.1 & 0.9 & 0.1 & 0.9 & 0.1 & .095 & NA & NA & NA \\
\hline
\end{tabular}

EAB: electrically assisted bicycle, SD: standard deviation, VO2: oxygen consumption, MET: metabolic equivalents, RPE: rate of perceived exertion, VE: minute ventilation, VCO2: carbon dioxide output, RER: respiratory exchange ratio. 
Figure 1

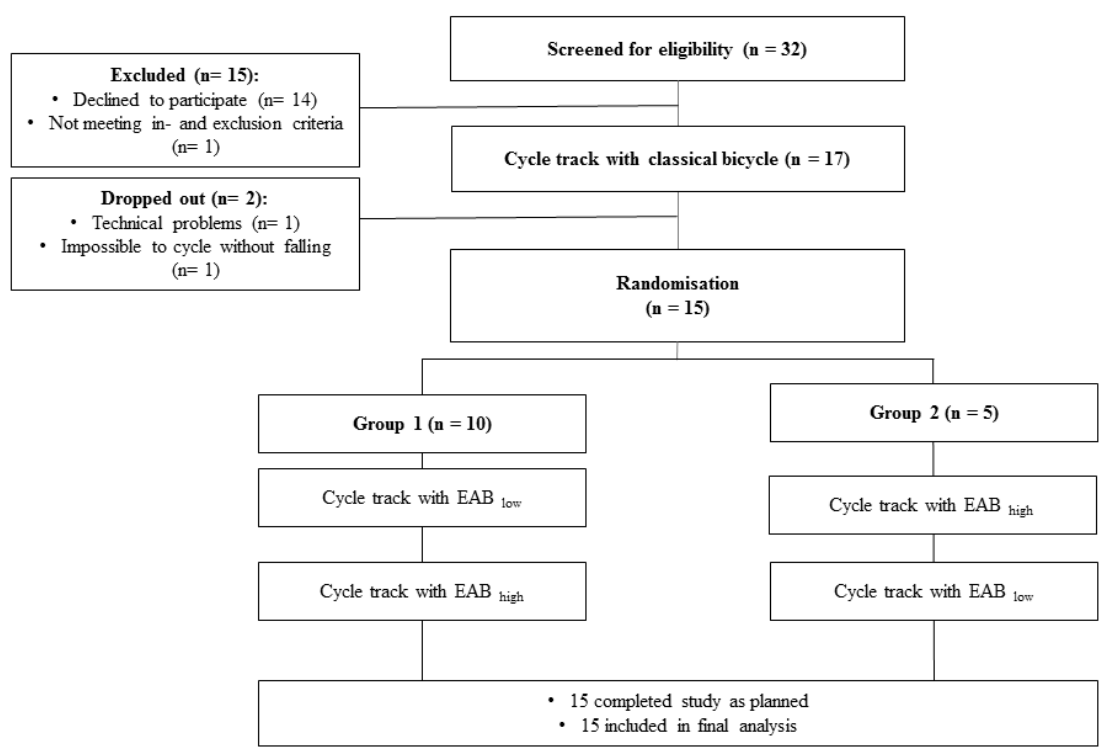


Figure 2

A.

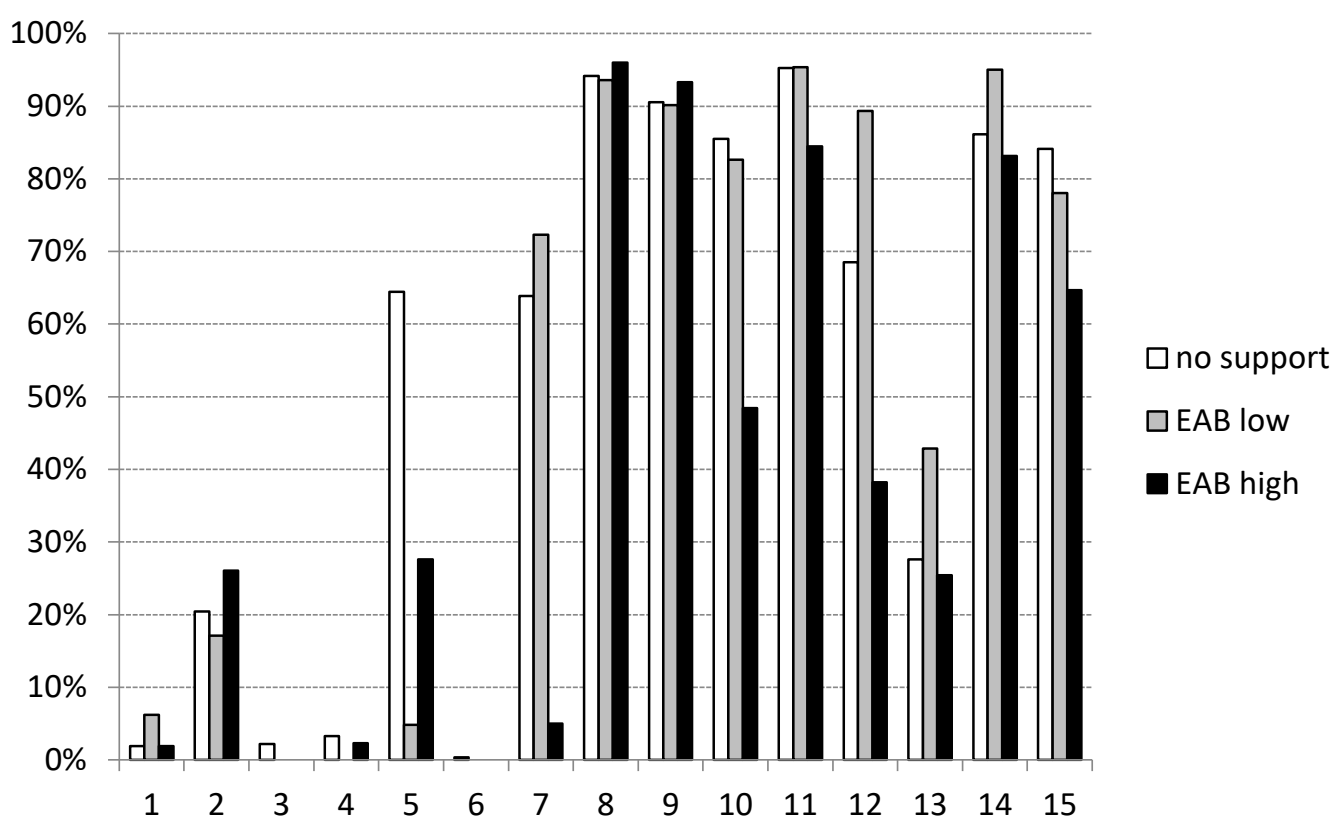

B.

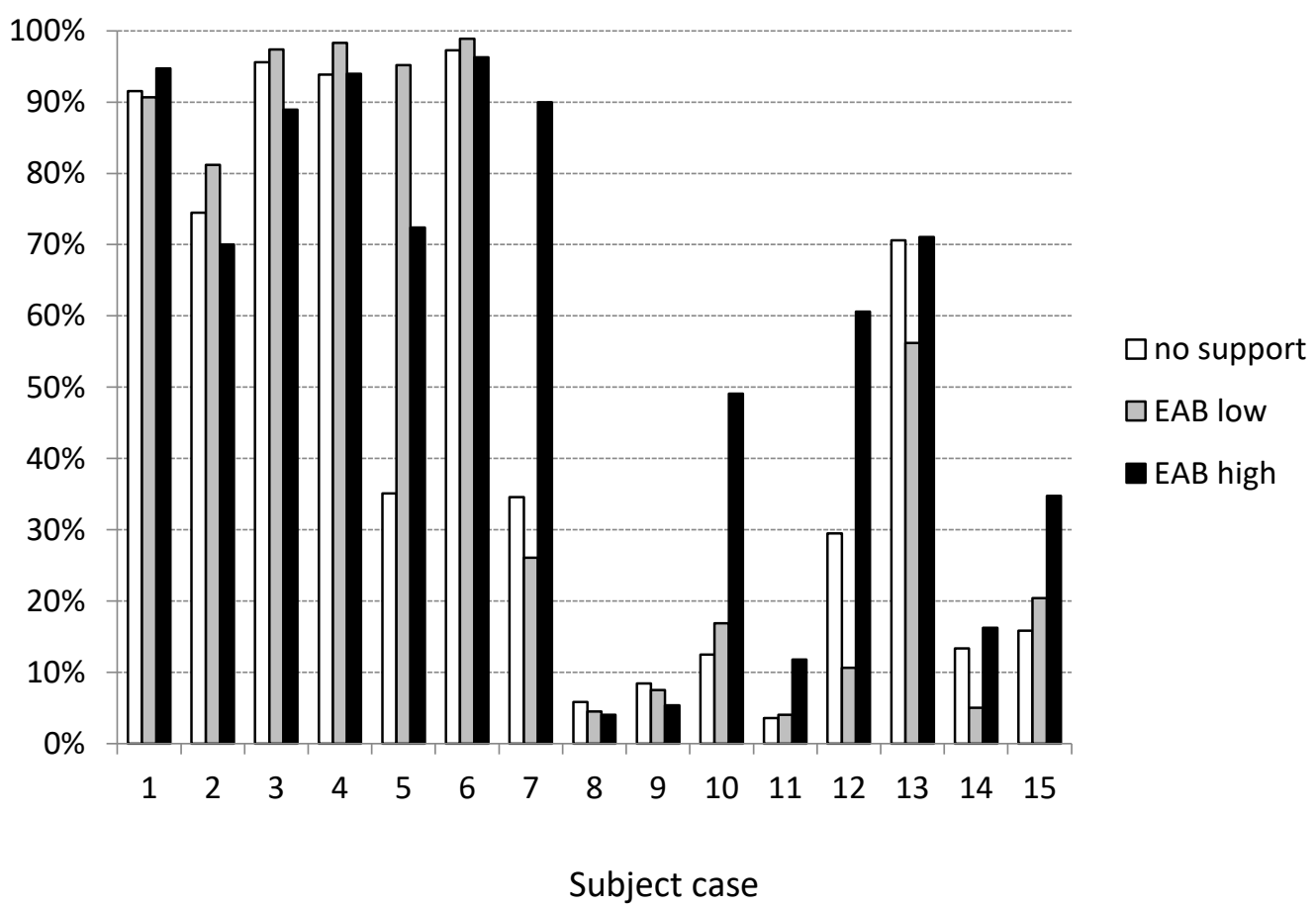

